\title{
Outlier-based Health Insurance Fraud Detection for U.S. Medicaid Data
}

\author{
Dallas Thornton ${ }^{1}$, Guido van Capelleveen ${ }^{1}$, Mannes Poel ${ }^{2}$, \\ Jos van Hillegersberg ${ }^{2}$ and Roland M. Mueller ${ }^{3}$ \\ ${ }^{1}$ University of California, San Diego, San Diego Supercomputer Center, \\ 9500 Gilman Dr., MC 0505, La Jolla, CA 92093, U.S.A. \\ ${ }^{2}$ University of Twente, Drienerlolaan 5, 7522 NB Enschede, The Netherlands \\ ${ }^{3}$ Berlin School of Economics and Law, Badensche Straße 52, 10825 Berlin, Germany
}

Keywords: Fraud Detection, Medicaid, Healthcare Fraud, Outlier Detection, Anomaly Detection.

\begin{abstract}
Fraud, waste, and abuse in the U.S. healthcare system are estimated at $\$ 700$ billion annually. Predictive analytics offers government and private payers the opportunity to identify and prevent or recover such billings. This paper proposes a data-driven method for fraud detection based on comparative research, fraud cases, and literature review. Unsupervised data mining techniques such as outlier detection are suggested as effective predictors for fraud. Based on a multi-dimensional data model developed for Medicaid claim data, specific metrics for dental providers were developed and evaluated in analytical experiments using outlier detection applied to claim, provider, and patient data in a state Medicaid program. The proposed methodology enabled successful identification of fraudulent activity, with 12 of the top 17 suspicious providers $(71 \%)$ referred to officials for investigation with clearly anomalous and inappropriate activity. Future research is underway to extend the method to other specialties and enable its use by fraud analysts.
\end{abstract}

\section{INTRODUCTION}

Roughly $\$ 700$ billion of the $\$ 2.7$ trillion spent on healthcare in the US is attributable to fraud, waste, and abuse (Kelley 2009). Healthcare payers deal with fraudulent practitioners, organized criminal schemes, and honest providers who make unintended mistakes while billing for their legitimate services. Government programs are particularly susceptible to fraud, as it is harder to exclude problematic providers than in privately managed provider networks. Data analysis methods utilized in other sectors are not yet widely deployed and utilized in this domain, partially due to the high level of subject matter knowledge needed to adapt these techniques to the unique environments in which they must be deployed. Yet, with up-front engineering and ongoing adaptations, techniques such as outlier detection offer a lifeline to programs struggling to rein in spiraling costs and remain solvent.

Unsupervised data mining techniques such as outlier detection are suggested as effective predictors for fraud. This paper proposes and evaluates a method for applying outlier detection to healthcare fraud based on comparative research, fraud cases, and literature review. Based on a multidimensional data model developed for Medicaid claim data (Thornton et al. 2013), specific metrics for dental providers were developed and evaluated in analytical experiments using outlier detection applied to claim, provider, and patient data in an actual state Medicaid program. The proposed methodology successfully identified fraudulent activity, with 12 of the top 17 suspicious providers $(71 \%)$ referred to officials for investigation with clearly anomalous and inappropriate activity. The research methodology of Hevner et al. (2004) was chosen to guide our work of designing a construct for fraud detection that should be improved and adapted based on environmental feedback and an evolving knowledge base.

\section{KNOWLEDGE BASE}

Existing literature discusses how electronic fraud detection could help combat health care fraud by 
securing the claim input process, checking on irregularities, and analyzing claim data sets to search for behavioral indicators of fraud (Aral et al. 2012; Bolton \& Hand 2002; Forgionne et al. 2000; Ortega et al. 2006). Unfortunately, due largely to the begrudging acknowledgement of fraud in health care, the complexity of the claim systems, the size and distributed storage of claim data and the late and relatively low funding for fraud detection, development of electronically fraud detection systems is lagging industries such as banking and telecommunications. There is a large base of statistical methods that are also used in other industries and could potentially be applied within the health care industry (Travaille et al. 2011). Some research reported specific fraud scheme detection using data mining approaches (Forgionne et al. 2000; Major \& Riedinger 2002; Musal 2010; Ng et al. 2010; Shin et al. 2012), however an outstanding challenge is to explore other healthcare fields for potential data mining possibilities and develop a more applied approach to this problem.

Data mining is gaining more attention by researchers as a potential tool to find healthcare fraud more easily (Aral et al. 2012). Most of the studies consider outlier detection as one of the primary tools (Weng \& Shen 2008). Researchers have combined multiple methodologies such as fuzzy logic in medical claims assessment and neural networks for automatic classification (Travaille et al. 2011). In the early 2000's, some initial concepts of data warehousing for data mining purposes in health care arose (Forgionne et al. 2000). Major and Riedinger (2002) developed an electronic fraud detection application to review providers on 27 behavioral heuristics and compare those to similar providers. A provider score was calculated based on these heuristics followed by a frontier identification method to select providers as candidates for investigation. Another example identified a number of meaningful rare cases in pathology insurance data from Australia's Health Insurance Commission using an on-line discounting learning algorithm (Yamanishi et al. 2004). In Taiwan scientists developed a detection model based on process mining that systematically identified practices derived from clinical pathways to detect fraudulent claims (Yang \& Hwang 2006).

In Canada, researchers used Benford's Law Distributions to detect anomalies in claim reimbursements ( $\mathrm{Lu} \&$ Boritz 2005). Although the method did find some suspicious behavior, its potential for fraud identification seemed to be limited in this case. One of the main reasons is that
Benford's law uses overly frequently used firstdigits to find fraud. However, this does not necessarily apply to services with payer-fixed prices.

In Chile, a private health insurance company built applications of neural networks used to find medical abuse and fraud (Ortega et al. 2006). The innovative aspects of the application concerned a method that could process the claims on a real time basis. Other examples are the application of association rule mining to examine billing patterns within a particular specialist group to detect these suspicious claims and potential fraudulent individuals (Shan et al. 2008) or the use of clustering procedures as well as regression models for geographical analysis of possible fraud (Musal 2010).

$\mathrm{Ng}$ et al. (2010) experimented on detecting noncompliant consumers (prescription shoppers) in spatio-temporal health data of Medicare Australia using multiple metrics that flagged providers. Although beneficial experimental results were achieved and the authors consider spatial and temporal factors to be effective in metrics, significant benefits concerning the use of spatialtemporal factors instead of more traditional metrics could not be verified. The simpler metrics, such as multiple visits or prescription percentages of pharmacy visits for drugs of concern, have proved valuable activity as well. Also Tang et al. (2011) described the problem of prescription shopping in their research. They used integrated techniques like feature selection, clustering, pattern recognition and outlier detection. Using a threshold on the outlier score provider groups could be marked as potential fraudulent.

Iyengar et al. (2013) described a methodology for identifying and ranking candidate audit targets from prescription drugs. The researchers developed a normalized baseline behavioral model for each prescription area and searched for statistically significant deviations from that model. For some of the areas, up to 500 features were used to find anomalies. For the narcotic analgesics drug class, all the known cases of fraud were correctly identified by the model as being very abnormal and excessive.

The research of Thornton et al. (2013) builds upon Sparrows fraud type classifications and developed a Medicaid multidimensional data schema and elaborated on analysis techniques that help to predict the likelihood of finding fraudulent activities.

A scope and extent of health care fraud was described by Travaille et al. (2011) that provided an overview of the electronic fraud detection from other industries applicable to the health care industry. The 
authors advocated the use of statistical methods for detection fraud and abuse for many of the health care areas, and gave insight in the multiple fraud schemes that are used by criminals in health care.

The work of Phua et al. (2010) includes a comprehensive survey of data mining-based fraud detection research. He categorizes, compares and summarizes a decade of research papers about automated fraud detection.

In general, the papers suggest and justify the applicability of data mining techniques in detecting healthcare fraud. Most describe the process of metric gathering, valuing and validation, and how dynamics force adaptation within a continuously changing environment. Most papers have a focus on a specific health care area, which seems to indicate a nonhomogeneous field for application. In search for generalizability we look for a common approach that can be extended and applied flexibly at scale. Our goal is to apply this methodology on the total set of Medicaid data, including over 70 million beneficiaries. Therefore, we need a generic approach to developing predictors for detection of healthcare fraud in multiple health specialties.

\section{ENVIRONMENT}

"Fraud is the intentional deception or misrepresentation that an individual knows to be false or does not believe to be true and makes, knowing that the deception could result in some unauthorized benefit to himself/herself or some other person" (Department of Health and Human Services 1998). In developing our metrics, model, and overall construct, we bear in mind this definition and set the goal for identifying provider-based fraudulent activity. Providers are the initiating actor for billing healthcare payers, and, as such, quickly become the nexus for fraud schemes.

When a provider participates in Medicaid, the provider agrees to reimbursement by the state and submits claims for payment directly to the state or managed care entity. States operate claims processing systems that perform various prepayment checks and edits to inspect the claim's legitimacy. Edits and audits verify information with honest providers in mind, but they are not designed to detect fraud schemes of any depth (Sparrow 2000). These systems simply cannot verify that the service was provided as claimed, that the diagnosis is correct, or whether the patient is even aware of the claimed services.

Health care reimbursement policy varies state to state and even across different patient populations, meaning metrics and predictors of fraud in one state must be adapted to be relevant in the differing policies of another. In this paper, we focus on a generalizable model that can be applied across programs with differing parameters and settings applied to accommodate for policy and program differences.

\section{METHOD FOR APPLYING OUTLIER DETECTION TO HEALTHCARE FRAUD}

To best address the need for iterative metric review, adjustment processing, and iterative weighting modifications, we have developed an iterative process for applying outlier detection to healthcare fraud, shown in Figure 1. In the subsequent subsections, we will describe each phase of this process.

\subsection{Compose Metric Sets for Domains}

Metrics can be derived and designed in multiple ways: through case analysis, by literature review, by study of attributes in the data model, or by cooperation with businesses of an industrial sector. Although a case study may be an instrument that helps to create a set of metrics, evaluation of the metrics by means of experts and flagging results is an absolute necessity. The set of metrics chosen in this paper consists of metrics based on analyzed cases from the FBI (U.S. Federal Bureau of Investigation 2013), metrics developed through discussions with healthcare fraud experts, and metrics found in existing literature (Musal 2010; $\mathrm{Ng}$ et al. 2010; Shin et al. 2012; Tang et al. 2011; U.S. Government Accountability Office 2012). To understand the process of fraud metric extraction we illustrate two examples of fraud cases that helped to design identifying metrics.

First, in a recent fraud case in New Jersey, a physician and owner of a home-based physician services firm for seniors plead guilty for charging lengthy visits to elderly patients that they did not receive (District of New Jersey U.S. Attorneys Office 2013). The physician in dispute received at least half a million dollars and was eventually detected because he became the highest billing home care provider among over 24,000 doctors in New Jersey from January 1, 2008 through October 14, 2011. Intentional over-billing for services, also 
known as up-coding, is a typical behavior that can be detected using metrics. Derived from this case was the 'visit length' metric. The comparison of peers based on the proportion of each visit length could identify such fraud. The assumption is that criminal providers may claim a higher proportion of lengthy patient visits than their peers.

Second, a Texas doctor owned a community medical center and falsely represented office visits and diagnostic test that were medically unnecessary from February 2010 until February 2011 (District of Texas U.S. Attorneys Office 2013). In exchange for submitting to diagnostic tests, patients at the clinic were prescribed controlled substances, incentivizing the patient to return for follow-up visits. The creation of fictitious symptoms provided the doctor reason to prescribe the narcotics and provided plausible rationale for ordering more tests. An indication for this type of fraud can potentially be found in the referral rate. Despite their apparent deteriorating condition, patients in this case would rarely be referred to an outside specialist, as this would obviously reveal that they are healthy. It might also be found in the amount and/or types of specific tests the doctors would prescribe to their patients as compared to peer physicians. A third telling metric could be patient retention and frequency of visits. Although most general physicians have returning patients, when a large proportion of patients return too often, this would seem quite suspicious and stand out as compared to peers.

Metric identification is a complex task that requires the knowledge of both, the health care domain and statistical theory. In the metric design process, it requires more than analysis of fraud cases to find fraud indicators. A group of outliers will normally consist of some outliers based on statistical deviation, just by chance, which cannot be filtered within a single metric. Only when fraudulent providers will take a more deviant position in the group of outliers, normal providers may shift to the non-outlying group, leaving the 'bad guys' separated. However, there are always providers that will be classified as outliers, although they are not fraudulent, due to the fact that their practice actually differs too much from the closest comparable peer group. Filtering this non-fraudulent provider or moving them to alternate peer groups can be done, but it is difficult to impossible without significant understanding of the domain.

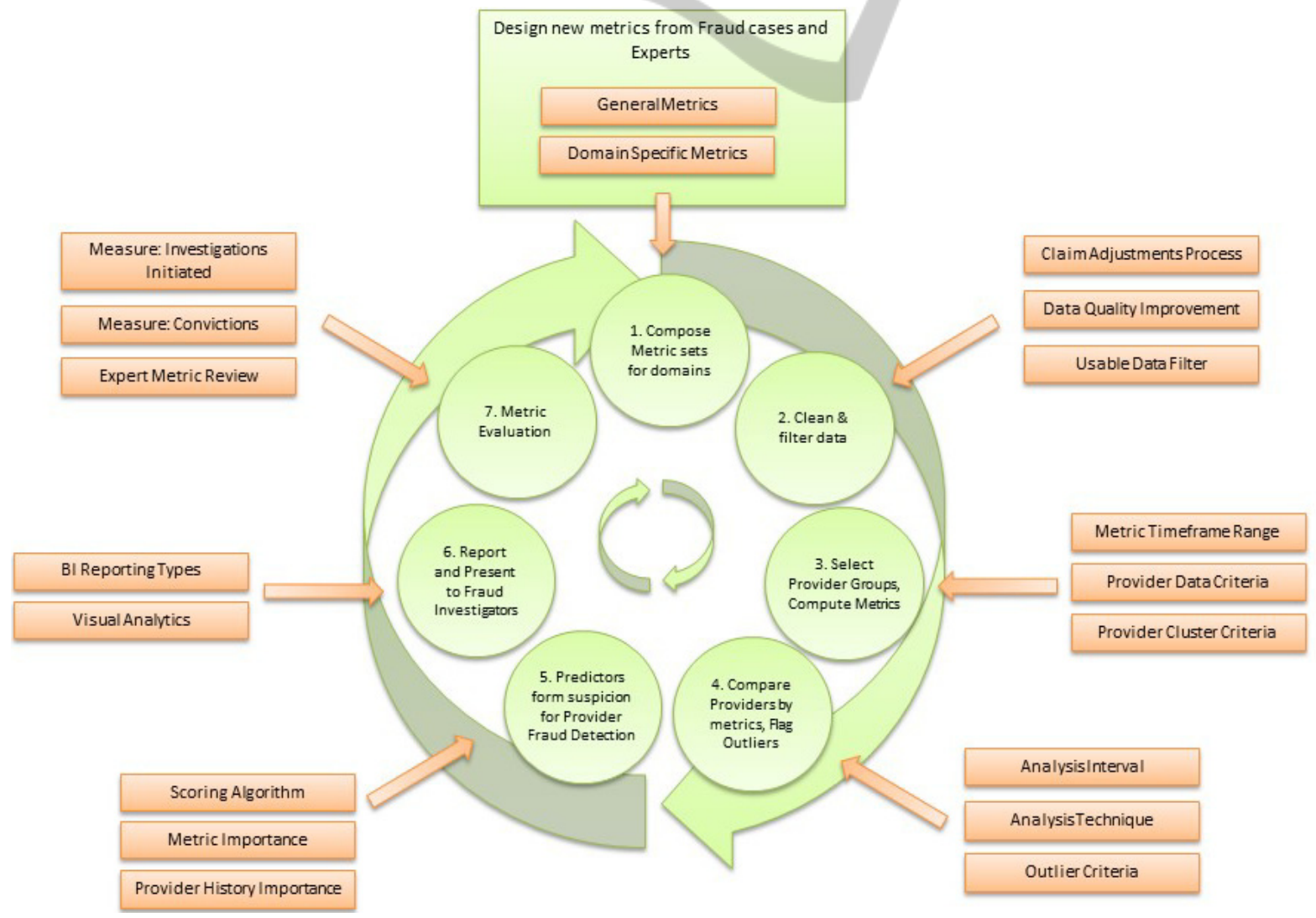

Figure 1: Method for Applying Outlier Detection to Healthcare Fraud. 
The set of metrics does not necessarily have to be large; on the contrary, often 25 to 30 features are sufficient. If hundreds of metrics have to be designed, the absolute amount of outliers is increased as well, which eventually will result in all providers displaying outlying behavior for some metrics. Metric identification is dependent on fraud experts and is an iterative process to find a set of metrics that works effectively. For our case study, we initially developed over 100 behavioral metrics. This list was subsequently refined to fifteen that could be applied to a relatively homogenous provider pool in the dental domain, feasible for implementation within our research case constraints.

\subsection{Clean and Filter Data}

This phase creates a workable set of data for the analysis. This consists of cleaning the data set and selecting only the relevant data of those providers to be analyzed.

The first task relates to data quality, which has to be estimated in order to determine the precision of computations. Where data quality may be reduced by multiple influences, three main concerns are addressed. First, merging multiple databases of information about common entities is frequently encountered in large commercial and government organizations (Hernández \& Stolfo 1998). Second, there is also the problem of entered data quality. Health insurance data is subject to quality problems in various ways. Data entry is often done by hand, which is shown to be inaccurate in about $4.4 \%$ in cases on personal information, and even higher percentages when abstracting data (Colin et al. 1994). Third is the use of inaccurate data. Claims are often incorrectly submitted and adjusted afterwards. These claims should be removed if possible. Data cleansing is highly suggested prior to analysis. Data cleansing will process the data in order to detect and correct (or remove) corrupt or inaccurate records from the record set, table, or database.

After cleaning, filtering is required - the task of selecting only that data which can be used for analysis. All data containing missing values that cause the inability to calculate metrics, should be removed or estimated with imputation. Claims that are voided from the system will be filtered out from the data set used for analysis. The result set of claim transaction data should meet the ISO 8000 data quality criteria, as far as possible, before continuing the analysis.

\subsection{Select Provider Groups, Compute Metrics}

Providers should be similar so that it is meaningful to compare their behavior. The main problem is that the more homogeneous a providers group is, comparison may be better delineate true outliers, however, the sample size of providers will decrease as well. Three questions arise. Is there a minimum sufficing data quantity that should qualify a provider for the metric? For example, a provider with only 2 claims per month should probably be excluded. What is the minimum provider sample size acceptable to delineate outliers with reasonable certainty? A group of only 5 providers will likely not produce trustworthy comparisons. What provider characteristics can be used to group similar provider populations suitable for comparisons? Apart from operating within the same domain or sub-domain, other provider characteristics may influence the analysis, such as the provider size or volume of patients. If a cluster analysis is done to detect such differences, the cluster criteria would help identify these different groups.

To apply metrics in analysis, calculations of those are performed and stored. The data time frame over which the metric is calculated must be defined. In our experiment we took a snapshot of provider behavior for each metric in a time frame.

\subsection{Compare Providers by Metric, Flag Outliers}

The analysis interval, the frequency to compute the metrics and perform the analysis, should be defined. A reasonable approach in our environment, in which new data is loaded monthly, is to calculate the metrics on a monthly basis. We defined requirements for the computational resources and for the subject matter experts.

Next, the appropriate analysis techniques and/or outlier detection methods should be used for each of the metrics. Examples of analysis techniques used in our experiment were univariate analysis, multivariate analysis, time-series analysis, and box-plot analysis. The following outlier detection methods were used: deviation from regression model, deviation clusters, single deviations from clusters, trend deviations, and peak deviations, making use of both non-parametric and parametric deviations. In the figures $2-5$ we will show some examples of the different analyses.

Figure 2 shows the regression analysis between the total dollar amount reimbursed by Medicaid to a provider, and the number of reimbursed claims. The 


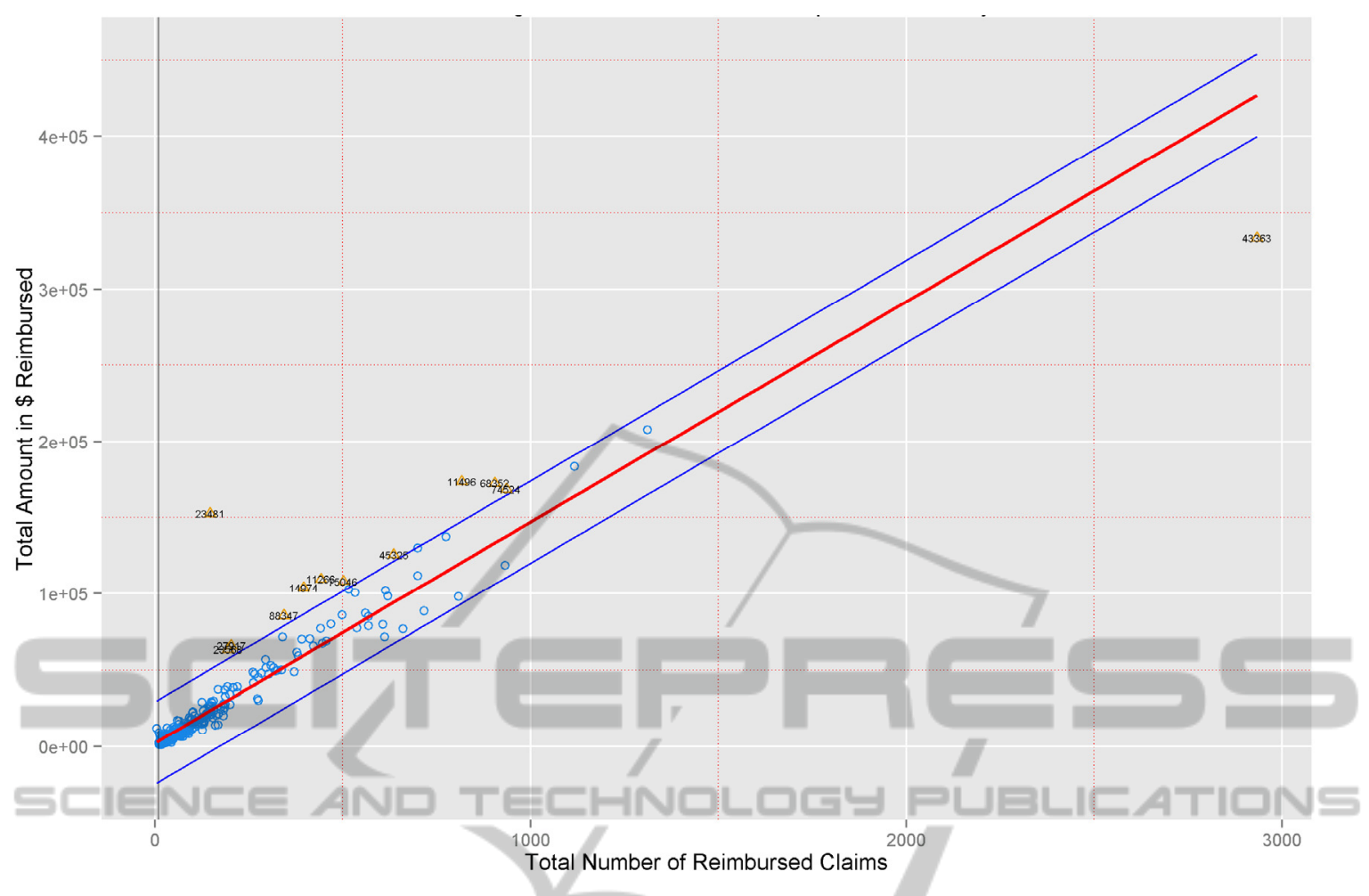

Figure 2: Regression of Reimbursement pre Beneficiary.

red line is the fitted linear model through the data points. The blue lines represent the 2.33 sigma's deviation from the logical model. Provider 23481, plotted in the left top corner, was one of the providers that attracted attention, because of its severe outlying behavior, and would be an interesting candidate for further analysis to find the cause of higher average of this provider on reimbursements.

Outliers influence sample means and deviations and therefore could mask themselves. This 'masking effect' could be reduced by robust estimation procedures (Rousseeuw \& van Zomeren 1990). However, we did not use these unmasking procedures in this study.

Figure 3 shows the boxplot outlier analysis by tooth code. Roughly 1-32 are adult teeth. The assumption is that providers that constantly perform treatments on the same tooth are interesting. Previous fraud cases mentioned examples of providers that claimed exact copies of procedures for different patients, to receive more reimbursements. These copies will be visible when we look at the tooth code, for example.

Another type of fraud that might reveal from this analysis is the recursive treatment on a tooth. If a provider fills it with amalgam, repeats it with a correctional procedure, but finally pulls it and puts a replacement, many procedures were performed on the same tooth. Sometimes this happens because of misdiagnoses, estimation errors, or coincidence. We took provider 42953 that almost claimed $20 \%$ of its procedures on code 03 , about a 140 procedures. To compare, the second most billed tooth of this provider appeared 27 times, all other teeth below 10 . The procedures are spread over multiple patients, in general one or two per patient. Most of the procedures have code D0120, a periodic oral evaluation on an established patient. The procedures are nicely spread over the whole year, around 12 per month.

Figure 4 shows the time series of a group of providers with outlying peaks. Those providers with a constant flow of claim submissions were compared to see if sudden increases in claim numbers could be found. The black dots represent the outlying peak that has been identified. One interesting finding is the green line (42748). The provider is not claiming for patients and suddenly around week 13, he claims over 300 patients per week. Simple explanations might be the use of multiple Medicaid provider IDs, or a wrong registration of specialty, namely the provider corresponds to a mobile dental practice. 


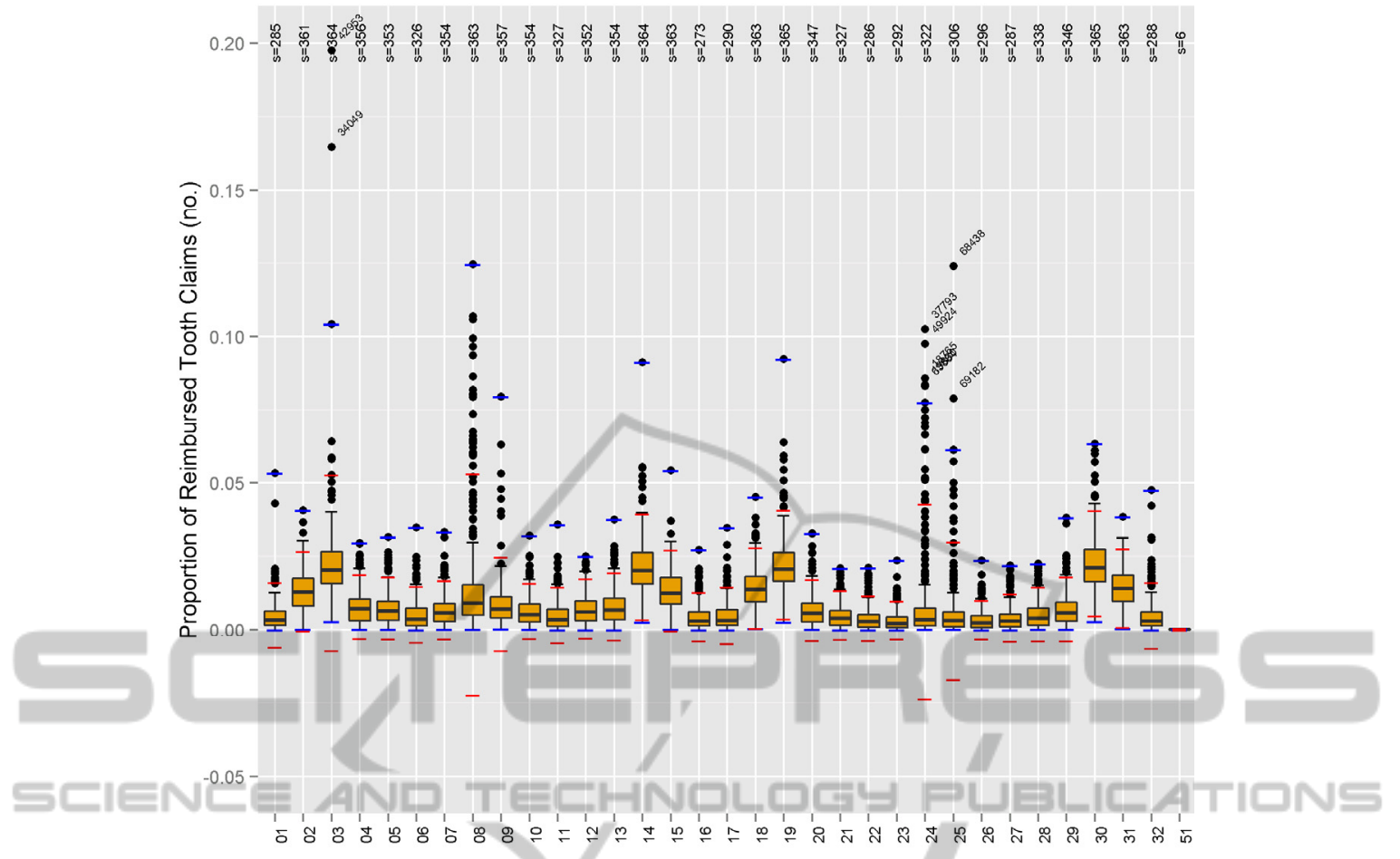

Figure 3: Tooth Code Analysis.

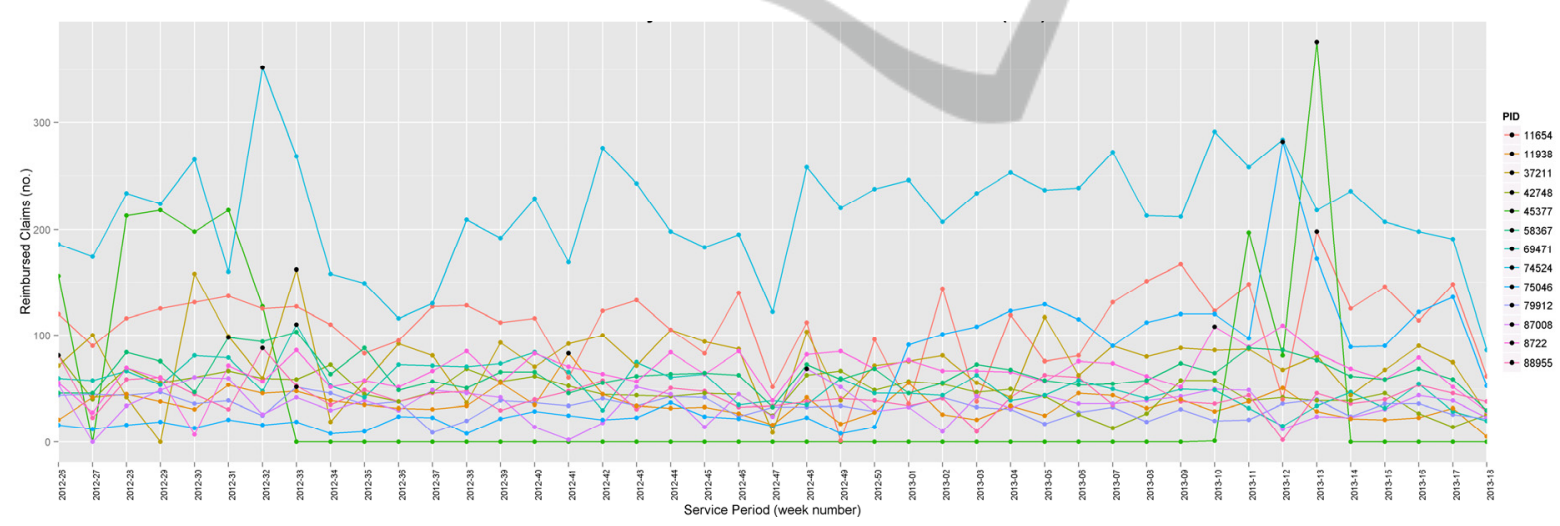

Figure 4: Peak Analysis: Time Series with Outliers of Reimbursed Claims.

Another provider that lighted up on this figure was provider 75046. Questionable is the sudden peak in week 12 of 2013. The number of claims rises from around a 100 claims per week, to almost 300 . After analysis on a service code level we found suspicious claims. Many children that visited the clinic that week received exactly the same treatments, only on different teeth. The combination of codes to reappear so frequently can't almost be a coincidence and should definitely be further investigated.

Figure 5 shows the combination of multivariate clustering and outlier detection. All data points exceeding the pink line represent 2.33 sigma's deviation in the extreme y direction from the cluster mean. Marked outliers thus have a considerable high average of reimbursed claims per beneficiary. Several reasons are possible for this phenomenon; not all related to fraud. There could be strict examination intervals to provide the best care, where many people will see the dentist on a regular basis, although this is costly. The maximization of the reimbursement under Medicaid could be another reason for a high average. Looking at provider 6950, an analysis at the claim level was done. We found that the high claiming behavior was caused by the decoupling of services over multiple claims. When 


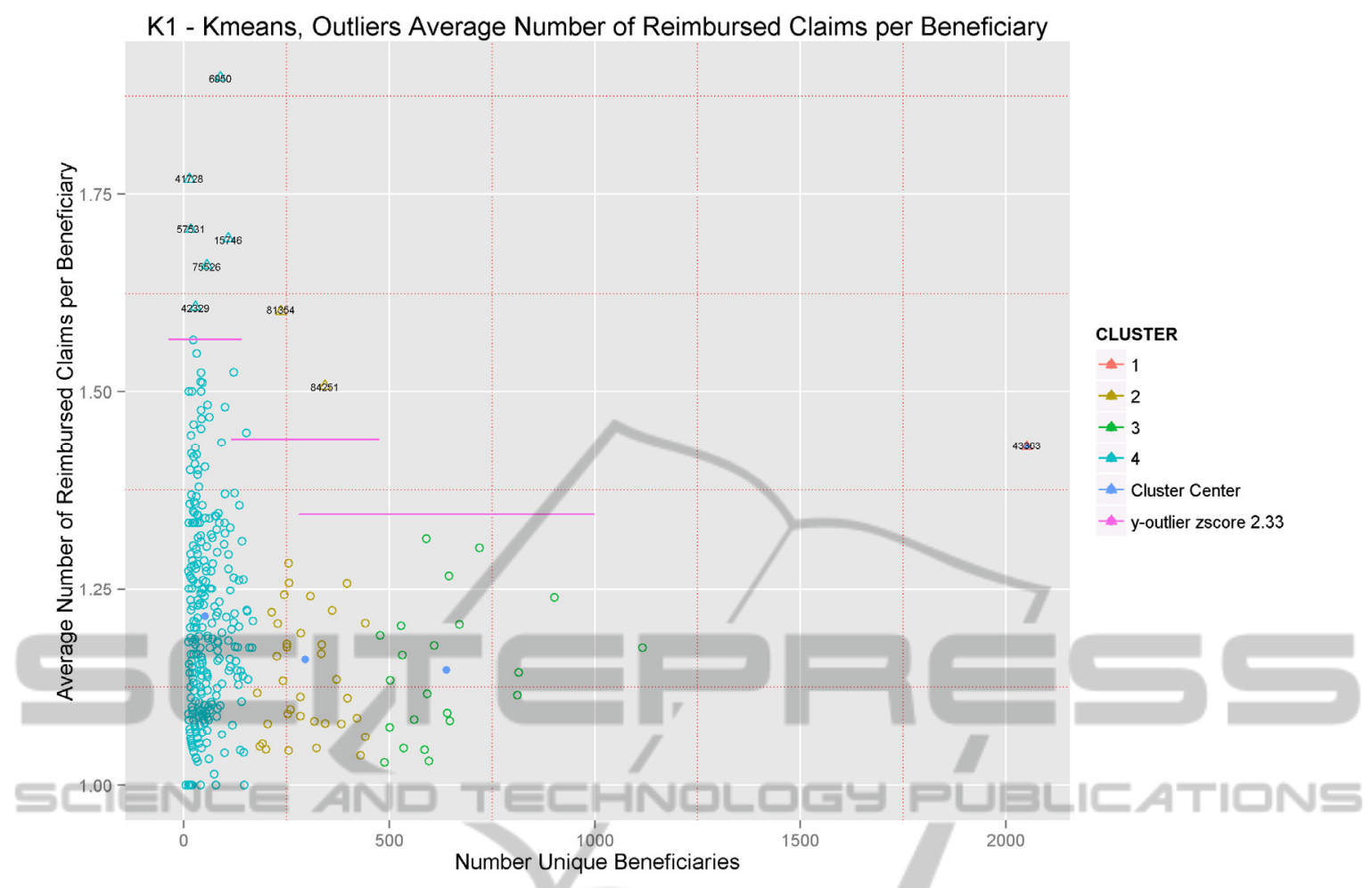

Figure 5: Multivariate Clustering and Outlier Analysis.

patient, service date, and treating dentists were identical, services were just send in as two claims. Does the provider decouple the claims to try to hide something, or is it an administrative error?

\subsection{Predictors Form Suspicion for Provider Fraud Detection}

An important aspect is how to report an anomaly and how to relate it to indication of fraud. Once an outlier criterion has been exceeded by a provider, we raise a flag for the provider in that period. A flag is the identification of an anomaly detected by the data mining algorithm. Scoring is the formula of the individual predictors for fraud detection based on the individual results of each of the outliers in provider analysis. The proposed scoring is a way of stacking suspicion. A single provider analytic will flag some honest providers. The assumption of using scoring to find the most interesting providers to investigate for fraud is that it will find those providers that are frequent outliers on multiple predictors.

\subsection{Report and Present to Fraud Investigators}

Fraud investigators need flexible reporting. In our view, there is no specific pre-defined way for reporting the data. A combination of dashboards and interactive multidimensional processing is therefore recommended. On the dashboard level we provide high level information on providers, by presenting the provider metric results, alerts on those providers that score deviant from others or on their history. Within comparative analysis, we enable investigators to drill down to the root of a claim to learn how deviations might have occurred and to collect the set of claims that needs further investigations. A list of alerts and their scores might be a starting point for investigators to begin their analysis.

\subsection{Metric Evaluation}

Evaluation of predictor effectiveness is required to make decisions for analyses and further metric developments. Measuring "success" is a difficult process since fraud is not established as fraud until after a litigation. Given the years of time lag, measuring convictions as justification for resource allocation and input for iterative improvement is suboptimal. Measuring investigations and audits initiated by fraud experts after internal review is certainly timelier and may be sufficiently reliable. If 
fraud investigation initiations are chosen as evaluation statistics, we may use the formulas of precision and recall to calculate the effectiveness of the method. A downside is that fraud investigations might be systematically wrong initiated distorting the effectiveness measurements. Fraud convictions might eventually provide the contrary evidence, however, we believe that fraud experts are capable of interpreting these measurements meaningfully. Thresholds, or configuration of the outlier detection algorithms influence the classification of data points as outliers. Restrictive outlier groups may minimize the number of potential fraud, while less restrained classification lead to false positives. The trade-off may be measured in terms of precision and recall (Aggarwal 2013).

\section{EVALUATION: MEDICAID DENTAL PROVIDERS CASE STUDY}

We performed a case study in which dental claims were analyzed on 14 different metrics. The seven stages to develop a successful fraud detection tool were followed and one iterative cycle was completed. A study on dental fraud has not been reported so far in literature, although it represents a large part of the Medicaid healthcare expenses. Dentistry is also a large and homogeneous group of providers that fit the criteria for the developed methodology well. An attempt was made in selecting metrics fitting within multiple known categories where fraud could be found.

\subsection{Data, Metrics, and Parameters}

For the case study, 11 months of Medicaid records from July 2012 until May 2013 were used for analysis, containing about 650.000 dental claims. The start date was the first of April 2013. To enhance peer group homogeneity, we chose to take only regular dental providers without any specialty as our provider group. Before calculation, data was processed for adjustments and cleaned for entries containing incorrect data such as null values, zero dollar payments, adjustments without original claims, and future servicing dates.

The metrics were computed using Oracle SQL Procedures and stored in database tables. Using the $\mathrm{R}$ language, scripts were developed to calculate and compare providers. The analyses developed in $\mathrm{R}$ made use of built-in functionality extended with statistical packages such a 'ggplot2' and 'cluster' required for functions such as fitting rule-based models, k-means algorithms, and boxplots. The application made use of a parameter file to configure the analysis by example set data filter criteria, outlier criteria, write back capabilities, and presentation characteristics.

The minimum requirements for analysis varied over the different types of analyses, as some metrics required larger data sets to achieve significant results. The baseline for a provider to be taken for analysis was set to a minimum of either 10 unique beneficiaries or $\$ 10,000$ reimbursed per month. Some of the predictors, such as the procedure code analysis, required a minimum service amount per year.

In the regression analysis, a deviation of 2.33 sigma from the underlying regression model was considered to be an outlier. In the univariate analysis we took the highest cluster, those providers that scored as a group the highest for the specific metric. In the multivariate analysis, using k-means clustering, outliers were defined by the outlier criteria, single data points deviating more than 2.33 sigma in y direction from its belonging cluster, or outlier cluster if smaller than 5 items. Clusters were formed using the k-means algorithm, set to 10 iterations. In the box-plots, the interquartile ranges defined the outlier criteria, configured for each metric separately.

As described in the methodology, a scoring mechanism is suggested for pointing out the most interesting cases to investigate. The scoring algorithm would make use of the number of flags, the importance value that has been assigned to a flag, and partly of the provider flagging history. In this study, the history was ignored due to the limited length of the data set, and, as an initial full cycle, flags for each of the metrics were equally weighted to evaluate their impact and relevance.

\subsection{Results}

Beginning with a data set of over 500 dental providers, the set was narrowed to roughly 360 providers through selection criteria. After performing the analysis, only 35 providers raised 2 or more of the potential 14 predictive flags. 17 providers raised 3 or more flags. We focused on these 17 providers to evaluate the potential efficacy of the approach.

We interviewed qualified healthcare fraud subject matter experts to evaluate the claims of and the raised flags by these 17 providers. While some of the 
flags could be understood as acceptable given the types of services rendered or due to the provider's operating environment, there was a preponderance of evidence suggesting that at least 12 of these 17 providers $(71 \%)$ with three or more flags should be immediately referred for audit and potentially to law enforcement.

\section{CONCLUSIONS AND FUTURE RESEARCH}

We structure our design science contribution according to the Hevner et al. (2004) framework and address a relevant problem in healthcare fraud detection. This paper offers an artifact and a description of a method for applying outlier detection to healthcare fraud along with an evaluation of this model in practice to a state-wide database of actual healthcare claims with over 500 providers. The model is evaluated by applying it in practice to actual healthcare data and having experts review the results of the analysis. The paper contributes to the literature by providing a roadmap for future applications of outlier detection in healthcare and potentially other corollary domains. We used the domain context of Medicaid and discussed considerations for its use in different data contexts. We communicated the model to stakeholders, including applying the overall process and specific scoring methods in practice.

Through this research, we learned many insights about antifraud efforts. Extensive healthcare subject matter expertise is required to design analysis techniques and interpret their results. Identifying 17 out of $360(5 \%)$ primary dental providers for further investigation, of which 12 of 17 (71\%) have been evaluated and deemed appropriate for formal investigation can be considered a successful outcome. As compared with prior comparative success rates of roughly $10 \%$ (Major \& Riedinger 2002), we see great opportunity in building upon this model in various ways. Future research will dive deeper, including evaluating specific outlier techniques relevant to different types of healthcare fraud, and look more broadly at methods and models for storing and preserving the scoring metadata and provenance information to allow for more automated scoring, model adaptability, and reconstruction. With this research we hope to both advance the state of the art in healthcare fraud detection and prevention, as well as materially assist tax payers and law enforcement in confronting this important societal challenge.

\section{REFERENCES}

Aggarwal, C. C., 2013. Outlier analysis, New York: Springer.

Aral, K. D. et al., 2012. A prescription fraud detection model. Computer Methods and Programs in Biomedicine, 106(1), pp.37-46.

Bolton, R. J. \& Hand, D. J., 2002. Statistical fraud detection: A review. Statistical Science, 17(3), pp.235-255.

Colin, C. et al., 1994. Data quality in a DRG-based information system. International Journal for Quality in Health Care, 6(3), pp.275-280.

Department of Health and Human Services, 1998. Medicare A/B Reference Manual - Chapter 21 Benefit Integrity and Program Safeguard Contractors. Available at: https:/http://www.novitas- solutions. com/refman/chapter-21.html [Accessed February 21, 2013].

District of New Jersey U.S. Attorneys Office, 2013. South Jersey Doctor Admits Making Half-a-Million Dollars in Fraud Scheme Involving Home Health Care for Elderly Patients. Available at: http://www.fbi.gov/ newark/press-releases/2013/south-jersey-doctoradmits-making-half-a-million-dollars-in-fraudscheme-involving-home-health-care-for-elderlypatients [Accessed March 28, 2013].

District of Texas U.S. Attorneys Office, 2013. Physician Pleads Guilty to Role in Health Care Fraud Conspiracy. Available at: http://www.fbi.gov/dallas/ press-releases/2013/physician-pleads-guilty-to-role-inhealth-care-fraud-conspiracy [Accessed March 1, 2013].

Forgionne, G. A., Gangopadhyay, A. \& Adya, M., 2000. An intelligent data mining system to detect healthcare fraud. In Healthcare information systems: challenges of the new millennium. Hershey PA: IGI Global, pp. 148-169.

Hernández, M. A. \& Stolfo, S. J., 1998. Real-world data is dirty: Data cleansing and the merge/purge problem. Data mining and knowledge discovery, 2(1), pp.9-37.

Hevner, A. R. et al., 2004. Design Science in Information Systems Research. MIS Quarterly, 28(1), pp.75-105.

Iyengar, V. S., Hermiz, K. B. \& Natarajan, R., 2013. Computer-aided auditing of prescription drug claims. Health Care Management Science, (July), pp.1-12.

Kelley, R. R., 2009. Where can $\$ 700$ billion in waste be cut annually from the US healthcare system? Ann Arbor, MI: Thomson Reuters, TR-7261 10/09 LW.

Lu, F. \& Boritz, J. E., 2005. Detecting fraud in health insurance data: Learning to model incomplete Benford's law distributions. In Machine Learning: ECML 2005. Springer, pp. 633-640.

Major, J. A. \& Riedinger, D. R., 2002. EFD: A Hybrid Knowledge/Statistical-Based System for the Detection 
of Fraud. Journal of Risk and Insurance, 69(3), pp.309-324.

Musal, R. M., 2010. Two models to investigate Medicare fraud within unsupervised databases. Expert Systems with Applications, 37(12), pp.8628-8633.

$\mathrm{Ng}$, K. S. et al., 2010. Detecting Non-compliant Consumers in Spatio-Temporal Health Data: A Case Study from Medicare Australia. In Data Mining Workshops (ICDMW), 2010 IEEE International Conference on. pp. 613-622.

Ortega, P. A., Figueroa, C. J. \& Ruz, G. A., 2006. A Medical Claim Fraud/Abuse Detection System based on Data Mining: A Case Study in Chile. In Proceedings of the 2006 International Conference on Data Mining. DMIN. Las Vegas, Nevada, USA: CSREA Press, pp. 224-231.

Phua, C. et al., 2010. A comprehensive survey of data mining-based fraud detection research. arXiv preprint arXiv:1009.6119.

Rousseeuw, P. J. \& van Zomeren, B. C., 1990. Unmasking Multivariate Outliers and Leverage Points. Journal of the American Statistical Association, 85(411), pp.633639.

Shan, Y. et al., 2008. Mining Medical Specialist Billing Patterns for Health Service Management. In Proceedings of the 7th Australasian Data Mining Conference - Volume 87. AusDM '08. Darlinghurst, Australia, Australia: Australian Computer Society, Inc., pp. 105-110.

Shin, H. et al., 2012. A scoring model to detect abusive billing patterns in health insurance claims. Expert Systems with Applications, 39(8), pp.7441-7450.

Sparrow, M. K., 2000. License To Steal: How Fraud bleeds america's health care system Updated., Boulder: Westview Press.

Tang, M. et al., 2011. Unsupervised fraud detection in Medicare Australia. In Proceedings of the Ninth Australasian Data Mining Conference-Volume 121. pp. 103-110.

Thornton, D. et al., 2013. Predicting Healthcare Fraud in Medicaid: A Multidimensional Data Model and Analysis Techniques for Fraud Detection. Procedia Technology, 9, pp.1252-1264.

Travaille, P. et al., 2011. Electronic Fraud Detection in the US Medicaid Healthcare Program: Lessons Learned from other Industries.

U.S. Federal Bureau of Investigation, 2013. FBI news blog. Available at: http://www.fbi.gov/news/ news_blog [Accessed April 18, 2013].

U.S. Government Accountability Office, 2012. Medicare Fraud Prevention: CMS has Implemented a Predictive Analytics System, but Needs to Define Measures to Determine its Effectiveness. Available at: http://www.gao.gov/products/GAO-13-104 [Accessed March 28, 2013].

Weng, X. \& Shen, J., 2008. Detecting outlier samples in multivariate time series dataset. Knowledge-Based Systems, 21(8), pp.807-812.

Yamanishi, K. et al., 2004. On-line unsupervised outlier detection using finite mixtures with discounting learning algorithms. Data Mining and Knowledge Discovery, 8(3), pp.275-300.

Yang, W.-S. \& Hwang, S.-Y., 2006. A process-mining framework for the detection of healthcare fraud and abuse. Expert Systems with Applications, 31(1), pp.56-68.

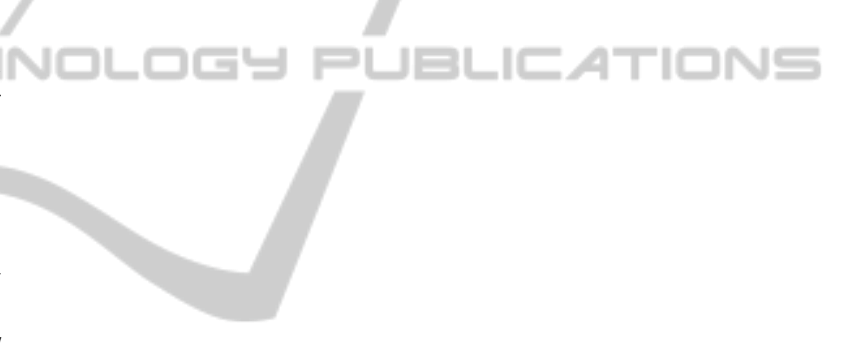

